\title{
Assessing Community Quality of Health Care
}

\author{
Jeph Herrin, Kevin Kenward, Maulik S. Joshi, \\ Anne-Marie J. Audet, and Stephen J. Hines
}

Objective. To determine the agreement of measures of care in different settings- - hospitals, nursing homes (NHs), and home health agencies (HHAs) — and identify communities with high-quality care in all settings.

Data Sources/Study Setting. Publicly available quality measures for hospitals, NHs, and HHAs, linked to hospital service areas (HSAs).

Study Design. We constructed composite quality measures for hospitals, HHAs, and nursing homes. We used these measures to identify HSAs with exceptionally high- or low-quality of care across all settings, or only high hospital quality, and compared these with respect to sociodemographic and health system factors.

Principal Findings. We identified three dimensions of hospital quality, four HHA dimensions, and two NH dimensions; these were poorly correlated across the three care settings. HSAs that ranked high on all dimensions had more general practitioners per capita, and fewer specialists per capita, than HSAs that ranked highly on only the hospital measures.

Conclusion. Higher quality hospital, HHA, and NH care are not correlated at the regional level; regions where all dimensions of care are high differ systematically from regions which score well on only hospital measures and from those which score well on none.

Key Words. Quality of care, community

Since 2005, hospitals have been reporting process of care data to the Centers for Medicare and Medicaid Services (CMS), which has used them to produce publicly reported measures of hospital quality of care. The number and specifications of these measures have changed over time; currently, CMS publicly reports process measures for four different conditions: acute myocardial infarction (AMI), heart failure (HF), surgical infection (SI), and pneumonia (PNE) (Hospital Compare 2014). Since 2009, CMS has also been reporting risk-adjusted 30-day readmission and mortality rates for AMI, HF, and PNE patients (Hospital Compare 2014). Starting in 2013, hospital Medicare reim- 
bursement was linked to hospital performance on these measures (VanLare and Conway 2012). As a consequence, a number of efforts, many successful, have been directed at improving hospital performance on these process and outcome measures.

Yet, while most of these efforts have focused on the hospitals as closed systems of care, hospitals are not isolated from the communities around them. There is a growing trend toward acknowledging this, particularly through the creation of accountable care organizations (ACOs), which are responsible for patient care across a range of settings. In addition, though CMS reports quality measures for nursing homes ( $\mathrm{NHs}$ ) and home health agencies (HHAs), there has been little examination of how these different types of health care work together at the community level.

Thus, to better identify and understand the differences between communities with high- and low-quality health care, we constructed metrics of the quality of hospital, HHA, and nursing home care using CMS's publicly reported quality measures. These quality measures have a number of limitations; they measure only specific aspects of care, rather than overall quality, and moreover, having been publicly reported for up to several years, providers may have found ways to perform well on these measures without improving overall quality. However, these measures are easily available, reported for almost all providers nationally, and are being used to inform policy and payment and thus have intrinsic relevance.

We aggregated these measures to the level of the hospital service area (HSA) and used them to construct metrics at the HSA level; we used these metrics to identify regions (HSAs) of measurably high quality on all dimensions, and compared these regions with regions that scored well only on hospital metrics, or scored poorly on all metrics. To validate the use of these metrics for identifying high-quality regions, we sought to identify systematic differences across these groups of regions; thus, as a secondary objective, we hypothesized that regions which scored well on all metrics would differ from those which scored well on only hospital measures or on no measures, when characterized with respect to sociodemographic status (SDS) and access to care.

Address correspondence to Jeph Herrin, Ph.D., Health Research \& Educational Trust, Chicago, IL; Yale University School of Medicine, New Haven CT, PO Box 2254, Charlottesville, VA 22902; e-mail: jeph.herrin@yale.edu. Kevin Kenward, Ph.D., Maulik S. Joshi, Dr.P.H., and Stephen J. Hines, Ph.D., are also with the Health Research \& Educational Trust, Chicago, IL. AnneMarie J. Audet, M.D., M.Sc., is with The Commonwealth Fund, New York, NY. 


\section{METHODS}

\section{Overview}

This was an observational study. We constructed aggregate metrics at the HSA level using health care quality measures from three sources: publicly reported hospital process and outcome measures from CMS Hospital Compare (HC) (Hospital Compare 2014); HHA quality measures from CMS Home Health Compare (HHC) (Home Health Compare 2014); and nursing home publicly reported quality measures from CMS Nursing Home Compare (NHC) (Nursing Home Compare 2014). Within each of these three care settings, we used factor analysis to reduce the measures to a smaller set of metrics. We examined the correlation among these metrics of quality of care and used them to identify regions that scored exceptionally high with respect to the full range of metrics considered, those which scored highly on only the hospital metrics, and those that scored well on none of the metrics. We summarized a number of SDS and care access characteristics of these different types of regions, and compared them across the three groups.

\section{Conceptual Model}

We assumed that the quality of health care in a region could be assessed using publicly reported measures of hospital, nursing home, and HHA health care quality in that region. Given the large number of hospital, nursing home, and HHA publicly reported measures, we further hypothesized that these could be combined and reduce to a more parsimonious set of metrics that would allow classification of regions. Given that regional variations in health care practice, including health care quality, are well established (Bernheim et al. 2010; Bradley et al. 2010; Laskey et al. 2010; Keating et al. 2012; Herrin et al. 2014), it is plausible that some regions would score highly in all three areas as a consequence of random distribution of quality metrics. Thus, we identified regions which scored well on metrics in all three care settings, those which scored well on only hospital quality metrics, and those which scored well in no area metrics. We used these categories to validate the concept of measuring the regional quality of health care; specifically, we hypothesized that if the metrics identified regions that were truly high quality in an underlying sense, they would differ systematically from those which scored well on only the hospital metrics. 
There are many ways in which HSAs can differ in ways that might affect or explain overall quality; for the purposes of validating the concept of measuring the quality of care of regions, we hypothesized specifically that differences would be reflected in regional differences in at least two domains of regional characteristics: sociodemographic factors and access to care.

First, much prior research has identified relationships between patient sociodemographics and quality of patient care. Patient SDS, income inequality, living alone, employment status, and educational level have been found to be associated with hospital outcomes, including hospital admission and mortality rates (Marcantonio et al. 1999; Tsuchihashi et al. 2001; Arbaje et al. 2008; Bradley et al. 2010; Howie Esquivel and Spicer 2012; Lindenauer et al. 2013; Shulan et al. 2013). Patient sociodemographic factors have also been found to be related to nursing home quality of care and HHA quality (Rosati et al. 2003; Mor et al. 2004; Smith et al. 2008; Li et al. 2011). More generally, SDS has been shown to influence the quality of physician care in the primary care setting (Franks and Fiscella 2002). There are several hypotheses regarding why these relationships exist: it may be that lower SDS patients receive lower quality care than other patients cared for by the same providers; it may be that low SDS is a marker for unobserved patient level risk factors that increase the likelihood of poor outcomes; or it may be that the quality of care in low SDS communities is generally lower than that in higher SDS communities. Separating these mechanisms is beyond the scope of this paper, but regardless of the causal path, it is plausible that the effects of disparities related to sociodemographic factors differ by care setting. Thus, we hypothesized that regional measures of sociodemographic factors would explain differences between regions that perform well on no metrics, those that perform well on hospital metrics alone, and those that also perform well on metrics in the other two care settings.

At the same time, we have previously found that access to care, measured by numbers of primary care and specialist physicians per capita, and the ratio of primary care to specialist physicians explained more of the regional variation in hospital readmissions than a large number of other health system and sociodemographic factors (Herrin et al. 2014); and it is plausible that the same measures of access to care will distinguish regions with higher quality care in nursing home and home health settings. Nursing home residents with access to more frequent or more timely physician care may be less likely to suffer infections, ulcers, or hospitalizations, and more likely to receive vaccines and medications, all metrics of nursing home quality. Less directly, HHAs, which often care for individuals with one or more chronic conditions, 
may find it easier to support those individuals if they have regular access to primary care. Thus, we hypothesized that increased access to care at the HSA level would explain some of the differences between regions which have lowquality care, those that have only high-quality hospital care, and those which have high-quality care in all three care settings.

Because the measures of quality and of regional factors are aggregated, this model is limited by the potential for false ecological inferences. That is, as we will not be able to link particular regional factors such as income to the individuals cared for by nursing homes-because an HSA has higher income we cannot conclude that the individuals in the nursing home have higher income - this model will not allow us to make conclusions about particular mechanisms of any relationships. However, if the model allows us to characterize the differences between low-quality HSAs, HSAs with high hospital quality, and those with high quality in all three care settings, it will provide insights into some determinants of high-quality care.

\section{Data Sources}

Hospital Service Areas. For our regional unit of analysis, we used HSAs. HSAs are collections of ZIP codes where most residents are served by the same set of hospitals; we used the HSA definitions developed and made publicly available by the Dartmouth Atlas of Health Care (The Dartmouth Atlas of Health Care 2014). While both larger and smaller regional units may be of interest, we used HSAs representing the largest regional unit where hospital, HHA, and nursing home quality of care might reflect community attributes; using larger regional units, such as hospital referral regions, would require aggregating larger numbers of patients and providers, which might mask meaningful variation in patient demographics and health system features.

Hospitals. Our study includes hospitals for which CMS publicly reported the following measures of hospital quality for 2010: seven outpatient (OP) measures and 34 measures of inpatient care for AMI, HF, PNE, and SI (Hospital Compare 2014); 30-day, risk-standardized readmission rates for patients discharged alive with AMI, HF, and PNE; 30-day, risk-standardized mortality rates for patients with AMI, HF, and PNE. These risk-standardized readmission and mortality rates use models that adjust for risk factors including age, comorbidities, and prior history, and are reported as six separate measures (Hospital Compare 2014). For each HSA, we calculated a pooled process met- 
ric as the total volume weighted average of all process measures in that group for all hospitals in the HSA. We also calculated HSA level AMI, HF, and PNE mortality and readmission metrics as the volume weighted averages of the corresponding measures of all hospitals in the HSA. For consistency with the other included measures, we reversed the readmission and mortality metrics, subtracting the rates from 1 so that higher measure value indicated higher quality.

Home Health Compare. We used data from Home Health Compare for 2010 (Home Health Compare 2014). Home Health Compare for 2010 reports for each HHA in the United States, the results of 21 different quality assessments. Assessments are made of patients cared for by each HHA; the complete methodology is available on the Home Health Compare website (Home Health Compare 2014). In addition to reporting the quality measures for each HHA, Home Health Compare also reports the full set of ZIP codes in which the HHA cared for patients during the measurement year. The full set of measures is reported in Table 2. These 21 measures were aggregated at the HSA level by averaging the reported values for all HHAs that served ZIP codes within the HSA, weighted by the number of ZIP codes served.

Nursing Home Compare. We used data from Nursing Home Compare for 2010 (Nursing Home Compare 2014). Nursing Home Compare for 2010 reports 18 different quality measures for each nursing home in the United States. Each measure is based on assessment of patients cared for by each $\mathrm{NH}$; the complete methodology is available on the Nursing Home Compare website (Nursing Home Compare 2014). In addition to reporting the quality measures for each $\mathrm{NH}$, Nursing Home Compare also reports the address, including ZIP code, where the NH is located. The full set of measures is reported in Table 3. These 18 measures were aggregated at the HSA level by averaging the values for all nursing homes located in the HSA.

Additional Data Sources. We used the Area Health Resource File (AHRF) from 2010 for population (2009 estimated); number of Medicare beneficiaries (2009 estimated); number of Medicaid beneficiaries (2009 estimated); number of general practitioners (2008 - this included both general practice and family medicine physicians); and number of specialists (2008 - this included all phy- 
sician specialists) (Area Resource File 2009-2010). We also used the Nielsen Pop Facts 2010 data file for ZIP code level data on median, average, aggregated and per capita income; numbers of households and families; and population (Pop Facts 2010). Because the AHRF contains observations at the county level, these were assigned to each of the ZIP codes in the county; ZIP code values from all sources were then averaged for each HSA, weighted by the population of the ZIP code.

\section{Statistical Analysis}

Approach. We used principal factor analysis to combine the 10 hospital, 21 $\mathrm{HHA}$, and $18 \mathrm{NH}$ measures into a smaller number of metrics for each setting. Within each set of setting (hospital, HHA, NH) measures, we performed principal factor analysis and used the number of eigenvalues greater than 1 to determine the number of independent factors (Guttman 1953; Kaiser and Rice 1974). We used this rule because, being conservative with respect to the number of factors identified (Nunnally and Bernstein 1994), it allowed us to select the most parsimonious set of factors with the most potential for distinguishing HSAs. Under the assumption that factors in each domain would be correlated with each other, we first examined the results of oblique rotation after factor analysis; if the resulting factors were only weakly correlated (Spearman's correlation $<0.4$ ), we then applied orthogonal rotation to identify which items loaded most on which factors; items which did not have a load of at least 0.4 on any factor were omitted from further analysis. Because fewer than half of hospitals and HSAs reported all 10 hospital measures, we used averages to construct the hospital metrics. For the nursing home and HHA metrics, we used the factor loadings for each item to create metrics using each of the retained items in each factor. We calculated Cronbach's $\alpha$ for the items in each factor.

We then summarized the final set of metrics and calculated the correlation between each pair. We then scored each HSA according to whether it was in the top quintile on each metric and ranked the HSAs according to number of metrics for which they were in the top quintile. HSAs which were in the top decile on this ranking were categorized as "high-quality" HSAs; those which were in the top decile of this ranking using only hospital metrics were "highhospital quality"; and those which were in the bottom decile on all rankings were "low-quality" HSAs. We chose to group HSAs by decile to have adequate numbers to be meaningful while minimizing heterogeneity within each group; in sensitivity analysis, we also used quintiles. Finally, we compared 
HSAs in these three mutually exclusive groups on HSA demographics and measures of access to care using $F$-tests based on ANOVA regressions.

Software. All analyses were performed using Stata 13.1 statistical software (StataCorp, College Station, TX, USA).

\section{RESULTS}

\section{Sample}

The final sample included 3,436 HSAs which contained 4,617 hospitals with either process or outcome measures publicly reported in 2010, which were served by $15,481 \mathrm{NHs}$ and 11,351 HHAs.

\section{Factor Analysis}

After inspecting the results of factor analysis and the content of the items, the seven hospital measures were grouped into three metrics - process, readmission, and mortality (Table 1). The $21 \mathrm{HHA}$ items were grouped into four metrics, as indicated in Table 2. These four metrics captured respectively: patient improvement ("Improvement"); timely or appropriate treatment of patients ("Treatment"); vaccine assessment ("Vaccines"); and routine checking or evaluation of patient health ("Checking"). The remaining items were not included

Table 1: Hospital Measures, Factor Loadings after Orthogonal Rotation, and Cronbach's Alpha for Final Metrics

\begin{tabular}{lcccr}
\hline Measure & Process & Readmission & Mortality & \\
\hline AMI mortality & -0.0785 & 0.0236 & $\mathbf{0 . 4 8 4 1}$ & 0.0656 \\
HF mortality & -0.0069 & -0.2399 & $\mathbf{0 . 5 5 5 9}$ & -0.0059 \\
PNE mortality & -0.0442 & -0.0131 & $\mathbf{0 . 5 9 7 6}$ & -0.0188 \\
AMI readmission & -0.0094 & $\mathbf{0 . 5 6 0 9}$ & -0.102 & 0.0383 \\
HF readmission & 0.003 & $\mathbf{0 . 6 5 9 9}$ & -0.0344 & 0.0154 \\
PNE readmission & 0.0102 & $\mathbf{0 . 6 4 5 9}$ & -0.0519 & -0.0338 \\
AMI process & $\mathbf{0 . 6 3 9 8}$ & -0.0526 & -0.0912 & -0.0934 \\
HF process & $\mathbf{0 . 7 1 4 1}$ & 0.0876 & -0.0415 & -0.0049 \\
PNE process & $\mathbf{0 . 7 2 7 6}$ & -0.008 & 0.0237 & 0.0609 \\
SI process & $\mathbf{0 . 7 3 9 4}$ & -0.0304 & -0.0149 & -0.0089 \\
Cronbach's alpha & 0.6628 & 0.7044 & 0.6006 & \\
\hline
\end{tabular}

Note. Bold entries indicate measure is assigned to that factor. 
Table 2: HHA Items, Factor Loadings after Orthogonal Rotation, and Cronbach's Alpha for Final Metrics

\begin{tabular}{|c|c|c|c|c|}
\hline Measure & Improvement & Treatment & Vaccine & Checking \\
\hline $\begin{array}{l}\text { How often the home health team began } \\
\text { their patients' care in a timely manner }\end{array}$ & 0.0172 & 0.2379 & -0.0053 & 0.322 \\
\hline $\begin{array}{l}\text { How often the home health team taught } \\
\text { patients (or their family caregivers) about } \\
\text { their drugs }\end{array}$ & 0.1309 & 0.5042 & -0.0266 & 0.393 \\
\hline $\begin{array}{l}\text { How often the home health team checked } \\
\text { patients' risk of falling }\end{array}$ & -0.0322 & 0.2362 & 0.0234 & 0.0792 \\
\hline $\begin{array}{l}\text { How often the home health team checked } \\
\text { patients for depression }\end{array}$ & 0.1229 & 0.2369 & 0.1481 & 0.6738 \\
\hline $\begin{array}{l}\text { How often the home health team } \\
\text { determined whether patients received a } \\
\text { flu shot for the current flu season }\end{array}$ & -0.0046 & 0.1029 & 0.8795 & 0.0224 \\
\hline $\begin{array}{l}\text { How often the home health team } \\
\text { determined whether their patients } \\
\text { received a pneumococcal vaccine } \\
\text { (pneumonia shot) }\end{array}$ & -0.0141 & 0.0657 & 0.8874 & 0.1209 \\
\hline $\begin{array}{l}\text { For patients with diabetes how often the } \\
\text { home health team got doctor's orders, } \\
\text { gave foot care, and taught patients about } \\
\text { foot care }\end{array}$ & 0.0162 & 0.8380 & 0.0546 & 0.1582 \\
\hline $\begin{array}{l}\text { How often the home health team checked } \\
\text { patients for pain }\end{array}$ & 0.1693 & 0.2112 & 0.0622 & 0.6826 \\
\hline $\begin{array}{l}\text { How often the home health team treated } \\
\text { their patients' pain }\end{array}$ & 0.0786 & 0.7475 & 0.0826 & 0.074 \\
\hline $\begin{array}{l}\text { How often the home health team treated } \\
\text { heart failure (weakening of the heart) } \\
\text { patients'symptoms }\end{array}$ & -0.0633 & 0.2736 & 0.0103 & 0.0987 \\
\hline $\begin{array}{l}\text { How often the home health team took } \\
\text { doctor-ordered action to prevent pressure } \\
\text { sores (bed sores) }\end{array}$ & 0.0645 & 0.8669 & 0.1104 & 0.0725 \\
\hline $\begin{array}{l}\text { How often the home health team included } \\
\text { treatments to prevent pressure sores (bed } \\
\text { sores) in the plan of care }\end{array}$ & 0.088 & 0.7118 & -0.0055 & 0.0825 \\
\hline $\begin{array}{l}\text { How often the home health team checked } \\
\text { patients for the risk of developing } \\
\text { pressure sores (bed sores) }\end{array}$ & 0.3425 & 0.1098 & 0.2261 & 0.5818 \\
\hline $\begin{array}{l}\text { How often patients got better at walking or } \\
\text { moving around }\end{array}$ & 0.6864 & 0.0578 & -0.2599 & 0.3119 \\
\hline $\begin{array}{l}\text { How often patients got better at getting in } \\
\text { and out of bed }\end{array}$ & 0.7576 & -0.0352 & 0.131 & 0.0346 \\
\hline How often patients got better at bathing & 0.7670 & 0.0779 & -0.0661 & 0.2356 \\
\hline How often patients had less pain when & 0.6862 & 0.1831 & 0.0396 & 0.0293 \\
\hline
\end{tabular}


Table 2: Continued

\begin{tabular}{|c|c|c|c|c|}
\hline Measure & Improvement & Treatment & Vaccine & Checking \\
\hline How often patients' breathing improved & 0.7756 & 0.0427 & 0.1693 & -0.1085 \\
\hline $\begin{array}{l}\text { How often patients' wounds improved or } \\
\text { healed after an operation }\end{array}$ & 0.2563 & 0.0242 & -0.3238 & 0.0804 \\
\hline $\begin{array}{l}\text { How often patients got better at taking their } \\
\text { drugs correctly by mouth }\end{array}$ & 0.7018 & 0.082 & -0.2273 & 0.2316 \\
\hline $\begin{array}{l}\text { How often home health patients had to be } \\
\text { admitted to the hospital }\end{array}$ & -0.4514 & -0.0215 & -0.3693 & -0.066 \\
\hline Cronbach's alpha & 0.8428 & 0.8839 & 0.9324 & 0.8149 \\
\hline
\end{tabular}

Note. Bold entries indicate measure is assigned to that factor.

because they did not load on any factor and had very minimal variation across HHAs. After inspecting the results of factor analysis and the content of the $\mathrm{NH}$ items, the 18 items were grouped into two metrics, as indicated in Table 3. These two metrics captured, respectively, the use of vaccines ("Vaccines") and patient pain ("Pain"). An additional item, percent of patients who received antipsychotic medication, had a loading of 0.4 on a third factor; this was substantially lower than the loadings of the other included items, and was not clearly aligned with quality of care, so we omitted it from further consideration. The remaining items did not load on any factors and had minimal variation across HSAs, so they were not included in subsequent analyses. Metrics were reversed if necessary so that positive direction indicated better outcomes for patients. Tables 1-3 also report the Cronbach's $\alpha$ for each of the hospital, HHA, and NH metrics; all were above 0.60 , and all but two were above 0.80 , indicating good internal consistency (Nunnally and Bernstein 1994).

\section{Dimensions of Quality}

Subsequent analyses are based on nine metrics of quality: three hospital metrics, four HHA metrics, and two NH metrics. Of the HSAs, 2,849 (83 percent) had data on all nine metrics; only 88 ( 3 percent) had data for fewer than seven metrics. The pairwise correlations among these nine - at the HSA level-are reported in Table 4 . The largest positive correlation across settings of care was a positive 25.9 percent between the HHA vaccine metric and the hospital readmission metric. The largest negative correlation across settings was negative 11.3 percent between NH pain with hospital mortality. Other correlations, and their statistical significance, are shown in Table 4. 
Table 3: Nursing Home Quality of Care Items, Factor Loadings after Orthogonal Rotation, and Cronbach's Alpha for Final Metrics

\begin{tabular}{|c|c|c|c|c|}
\hline Measure & Vaccine & Pain & Factor 3 & Factor 4 \\
\hline $\begin{array}{l}\text { Percent of long-stay, high-risk residents } \\
\text { with pressure ulcers }\end{array}$ & -0.1583 & -0.0412 & 0.1409 & 0.3604 \\
\hline $\begin{array}{l}\text { Percent of long-stay, low-risk residents } \\
\text { who lose control of their bowels or bladder }\end{array}$ & 0.0235 & 0.0158 & -0.3678 & -0.0342 \\
\hline $\begin{array}{l}\text { Percent of long-stay residents assessed and given, } \\
\text { appropriately, the pneumococcal vaccine }\end{array}$ & 0.7165 & -0.0384 & -0.0667 & -0.0497 \\
\hline $\begin{array}{l}\text { Percent of long-stay residents } \\
\text { assessed and given, appropriately, } \\
\text { the seasonal influenza vaccine }\end{array}$ & 0.5681 & -0.0067 & 0.3032 & -0.1291 \\
\hline $\begin{array}{l}\text { Percent of long-stay residents } \\
\text { experiencing one or more } \\
\text { falls with major injury }\end{array}$ & 0.079 & 0.2335 & 0.1848 & 0.0416 \\
\hline $\begin{array}{l}\text { Percent of long-stay residents } \\
\text { who have depressive symptoms }\end{array}$ & 0.0305 & 0.2877 & 0.1566 & 0.0412 \\
\hline $\begin{array}{l}\text { Percent of long-stay residents } \\
\text { who have/had a catheter } \\
\text { inserted and left in their bladder }\end{array}$ & -0.0135 & 0.1282 & 0.0061 & 0.1911 \\
\hline $\begin{array}{l}\text { Percent of long-stay residents } \\
\text { who lose too much weight }\end{array}$ & 0.0108 & 0.1234 & -0.0065 & 0.2956 \\
\hline $\begin{array}{l}\text { Percent of long-stay residents who } \\
\text { received an antipsychotic medication }\end{array}$ & -0.1051 & 0.0503 & 0.4423 & 0.0422 \\
\hline $\begin{array}{l}\text { Percent of long-stay residents who } \\
\text { self-report moderate to severe pain }\end{array}$ & -0.0365 & 0.7170 & 0.042 & 0.0072 \\
\hline $\begin{array}{l}\text { Percent of long-stay residents who } \\
\text { were physically restrained }\end{array}$ & -0.0597 & -0.0026 & 0.0646 & 0.2345 \\
\hline $\begin{array}{l}\text { Percent of long-stay residents whose } \\
\text { need for help with daily activities } \\
\text { has increased }\end{array}$ & -0.0723 & 0.0888 & 0.1367 & 0.0975 \\
\hline $\begin{array}{l}\text { Percent of long-stay residents with a } \\
\text { urinary tract infection }\end{array}$ & 0.0426 & 0.1708 & -0.0131 & 0.305 \\
\hline $\begin{array}{l}\text { Percent of short-stay residents assessed } \\
\text { and given, appropriately, the } \\
\text { pneumococcal vaccine }\end{array}$ & 0.8543 & -0.0547 & -0.1347 & 0.0373 \\
\hline $\begin{array}{l}\text { Percent of short-stay residents assessed } \\
\text { and given, appropriately, the } \\
\text { seasonal influenza vaccine }\end{array}$ & 0.8586 & -0.0046 & 0.0808 & -0.0088 \\
\hline $\begin{array}{l}\text { Percent of short-stay residents who } \\
\text { newly received an antipsychotic medication }\end{array}$ & -0.0793 & -0.0232 & 0.3727 & 0.111 \\
\hline $\begin{array}{l}\text { Percent of short-stay residents who } \\
\text { self-report moderate to severe pain }\end{array}$ & -0.098 & 0.6853 & -0.0412 & 0.0215 \\
\hline $\begin{array}{l}\text { Percent of short-stay residents with } \\
\text { pressure ulcers that are new or worsened }\end{array}$ & -0.0277 & 0.0486 & 0.1885 & 0.1936 \\
\hline Cronbach's alpha & 0.8195 & 0.7578 & & \\
\hline
\end{tabular}

Note. Bold entries indicate measure is assigned to that factor. 


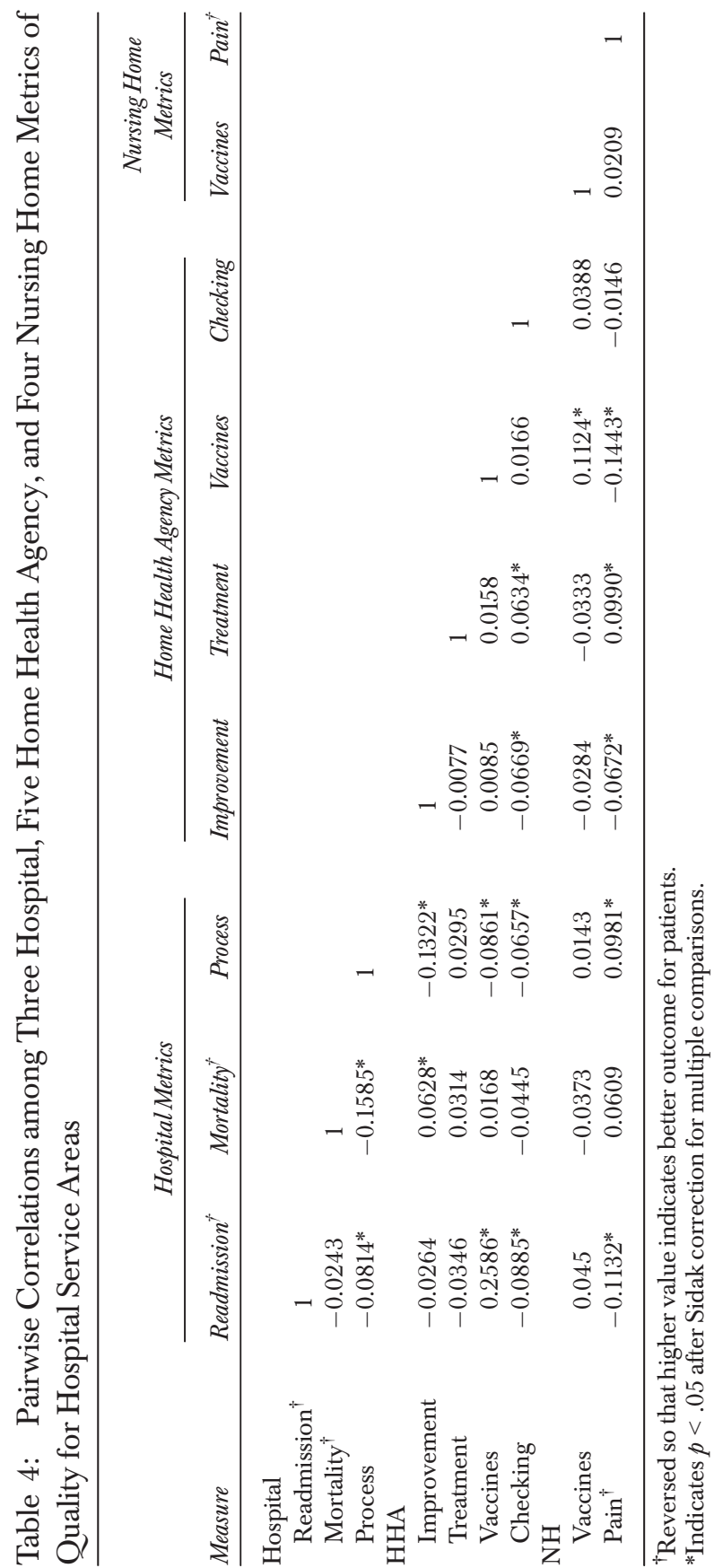


Table 5: Differences between HSAs Classified as High-Quality, High Hospital Quality, and Low-Quality Health Care Regions

\begin{tabular}{lcccc}
\hline & \multicolumn{3}{c}{ High Hospital } \\
& High Quality & Quality & Low Quality & Overallp \\
\hline$N$ & 108 & 183 & 473 \\
Population $(100 \mathrm{k})$ & $0.6(0.8)$ & $0.9(1.1)$ & $1.2(2.3)^{\dagger}$ & .004 \\
Families $(1 \mathrm{k})$ & $14.5(20.8)$ & $23.6(27.6)$ & $31.4(57.3)^{\dagger}$ & .002 \\
Households $(1 \mathrm{k})$ & $21.3(31.5)$ & $34.1(40.4)$ & $46.0(86.5)^{\dagger}$ & .003 \\
Medicare benes/1k & $17.7(5.1)$ & $17.3(4.4)$ & $17.7(4.4)$ & .620 \\
Medicaid benes/1k & $20.7(10.0)$ & $18.2(7.9)^{*}$ & $20.3(7.6)^{\star}$ & .007 \\
Aggregate income $(\$ 10 \mathrm{~m})$ & $15.8(27.4)$ & $26.3(31.4)$ & $30.9(61.8)^{\dagger}$ & .024 \\
Median household income $(\$ 10 \mathrm{k})$ & $47.5(16.0)$ & $54.2(20.1)^{*}$ & $46.9(12.0)^{\star}$ & .000 \\
Per capita income $(\$ 10 \mathrm{k})$ & $23.2(7.7)$ & $27.0(9.7)^{*}$ & $23.1(5.0)^{\ddagger}$ & .000 \\
Average household income $(\$ 10 \mathrm{k})$ & $59.9(21.4)$ & $69.4(26.9)^{*}$ & $58.8(13.8)^{*}$ & .000 \\
GPs/100k & $36.0(20.4)$ & $30.6(15.0)^{*}$ & $32.5(20.2)$ & .069 \\
Specialists/100k & $42.9(43.0)$ & $70.0(70.2)^{*}$ & $50.8(49.0)^{\ddagger}$ & .000 \\
GPs/specialist & $2.0(2.9)$ & $1.0(1.1)^{*}$ & $1.4(1.8)^{\dagger}$ & .000 \\
Hospitals (\#) & $12.0(16.2)$ & $5.2(9.6)^{*}$ & $6.0(10.4)^{\dagger}$ & .000 \\
NHs (\#) & $18.5(20.7)$ & $9.5(10.8)^{*}$ & $10.4(11.4)^{\dagger}$ & .000 \\
HHAs (\#) & $92.6(116.3)$ & $68.5(114.1)^{*}$ & $44.9(52.4)^{\dagger *}$ & .000 \\
\hline
\end{tabular}

*Contrast of High Quality and High Hospital Quality has $p$-value <.05.

${ }^{\dagger}$ Contrast of High Quality and Low Quality has $p$-value $<.05$.

\#Contrast of High Hospital Quality and Low Quality has $p$-value <.05.

\section{High- versus Low-Quality Regions}

There were 108 (3 percent) regions that were classified as "high-quality HSAs," 183 (5 percent) that were "high-hospital quality," and 473 (14 percent) that were "low-quality HSAs." Table 5 shows the results of comparing these three groups of HSAs. Among demographic characteristics considered, highquality HSAs had more Medicaid beneficiaries per capita, and lower aggregate, median household, average household, and per capita income $(p<.05)$; these differences were also significant between high-hospital quality and lowquality HSAs, and between high- and low-quality HSAs. Among the access to care characteristics considered, high-quality HSAs had higher numbers of GPs per capita, fewer specialists per capita, more GPs per specialist, more hospitals, more nursing homes, and more HHAs (all $p$ values $<.05$ ).

In sensitivity analysis in which HSAs were grouped by quintile rather than decile, results were similar, with the additional findings that GPs/100k varied significantly across the three groups $(p=.003)$; GPs and specialists per $100 \mathrm{k}$ differed significantly between low-quality and high hospital quality groups $(p<.05)$; and numbers of families and households differed significantly between high hospital quality and high-quality HSAs. 


\section{DISCUSSION}

In this analysis, we used publicly reported data to define and construct nine metrics of the quality of care at the community level, where community was defined as an HSA. The metrics measured quality in three care settings: hospital, nursing home, and HHA. We found that these nine metrics were largely uncorrelated with each other across the three settings; only one pairwise correlation across settings, of HHA vaccine use and hospital readmissions, had a magnitude greater than 20 percent. This indicates that high quality of one care setting is not often indicative of high quality of other settings in the same HSA, as measured by publicly available quality measures. In addition, we found that regions which had higher scores on all dimensions of quality differed systematically in demographics and access to care from those which only scored high on hospital quality metrics. This analysis demonstrates the feasibility of constructing metrics of community quality of health care that assess a broader range of quality than is captured by hospital measures alone; the systematic differences between high-quality HSAs and those with high hospital quality only suggests that the consideration of $\mathrm{HHA}$ and $\mathrm{NH}$ quality provides additional, meaningful, information about regional quality of care.

A number of prior studies have examined regional variations in the quality of hospital care for specific conditions (Bernheim et al. 2010; Bradley et al. 2010; Laskey et al. 2010; Keating et al. 2012), and we have previously examined variations in hospital readmission rates for all conditions (Herrin et al. 2014). However, by examining not only hospital care but also HHA and $\mathrm{NH}$ quality metrics, we have demonstrated that it is possible to identify regions that are high performing in all three settings, and identify attributes of these regions that set them aside from regions that perform well on only hospital metrics.

Importantly, the nine metrics we constructed represent related domains of care, and the correlations that do exist represent opportunities for improving the overall quality of care. That vaccines among HHAs is strongly associated with reduced readmission rates to hospitals in the same area suggests that efforts to increase the rates of vaccination among HHA patients might be an important factor in reducing readmissions, or at least that there are unobserved factors which contribute to both. Other relationships are less easily interpreted. The positive correlation between 
nursing home vaccine rates and HHA vaccine rates may be because both are influenced by local health care patterns, or may be because both are influenced by regional climate and consequent infection rates; the inverse relationship between reduced pain in nursing homes and HHA vaccine rates is even less easily interpreted.

Finally, in comparing the HSAs that were high performing in all three settings with those that scored well on all three hospital metrics (readmission, mortality, processes of care) on a range of several community factors related to SDS and health care access, we found that the most important differences related to access to primary care and specialty care, measured as the number of general practitioners per capita and the number of specialists per capita; these were 21 percent higher and 40 percent lower, respectively, in high-quality HSAs as compared to high hospital quality areas. While the more general finding is that quality of care in the three settings provides a signal not provided by hospital metrics alone, these specific differences are of intrinsic interest. They are consistent with our previous findings that GPs per capita were the strongest predictor of hospital readmission rates (Herrin et al. 2014), while pointing to a marginally stronger association with the quality of care in HHAs and nursing homes as well.

There are several limitations to this analysis. We used only a single year of data, 2010, and a single definition of community, the HSA, which means that our metrics are not necessarily valid for other years or geographical regions; however, our objective was not to produce a final index of metrics but to show that quality of care in the three settings provided signals beyond that provided by hospital care alone. Similarly, we did not attempt to compare high-, hospital, and low-quality HSAs on all characteristics that might be relevant; however, we selected factors that might influence quality of care in all three settings.

More critically, our metrics are derived entirely from CMS's publicly reported quality measures; as noted in the introduction, these may not reflect true underlying quality in any of the three care settings. Previous research has noted the weak relationship between process and mortality measures (Bradley et al. 2006; Werner, Bradlow, and Asch 2008); while consistent with our finding that these measures loaded on separate factors, this weak relationship also points to the challenges of making inferences about a single underlying hospital quality trait using only CMS's publicly reported measures. There is even less evidence that the measures for other care settings reflect true underlying quality, with some nursing home quality measures notably self-reported (Thomas 2014). This 
limitation of the CMS's publicly reported measures - that their reflection of true underlying quality across all care settings is likely imperfectimplies a corresponding limitation of our conclusions; that a region does well on the measures we included may in some part reflect that it is a region where attention is given to those measures, and that such attention is the true effect of, for example, higher SDS. However, without an objective "gold standard" of quality for any of the care settings, the CMS's publicly reported measures capture the best available information about the processes, outcomes, and standards of care that stakeholders, including patients and providers, have identified as important to highquality care.

A related limitation is that we included no measures of the quality of primary care. Given that prior evidence that such positive quality is related to SDS (Franks and Fiscella 2002), we would hypothesize that higher SDS regions would have higher quality primary care; higher densities of GPs could plausibly be related to either higher (due to competition) or lower (over supply) quality primary care. More important, the quality of primary care could mediate the effects of both SDS and access on quality of care in the three care settings we examined. Future study of the regional quality of care would benefit from standard measures of quality of primary care, perhaps through the measurement of ACOs.

Finally, as this is an observational study, we cannot make any causal inferences; for example, reduced HHA confirmed vaccine rates may lead to higher hospital readmission rates, or higher hospital readmission rates may occur in areas where HHAs are less attentive to vaccines because of differences in, for example, climate or demographics. However, we feel that this finding at least points to an area where further research might lead to improved quality of care for both HHAs and hospitals.

In summary, we identified nine distinct metrics of quality of care in the hospital, HHA, and nursing home settings; we found that they are poorly correlated at the regional level, as defined by the HSA, and used them to identify regions that are of high quality in all three settings. We identified a strong inverse relationship between HHA vaccine documentation and hospital readmission rates, as well as several other relationships across settings. Regions that perform well on the nine metrics differ in demographics and specialties of physicians from those that do well on only hospital metrics and from those HSAs that perform poorly on all of them. These findings provide evidence that the overall quality of care in communities can and should be assessed using quality measures from all three settings. 


\section{CONCLUSION}

Regional variations in quality of health care can be assessed using quality metrics constructed from publicly reported data from three settings of care: hospitals, nursing homes, and HHAs. The ways in which regions which perform well on measures in all settings differ systematically from other regions may inform efforts to improve quality of care across all settings.

\section{ACKNOWLEDGMENTS}

Joint Acknowledgment/Disclosure Statement: This work was supported by a grant from The Commonwealth Fund, New York, NY. We acknowledge the contributions of the anonymous reviewers for numerous helpful suggestions. None of the authors reported a conflict of interest with respect to this project.

Disclosures: Health Research \& Educational Trust is the publisher of HSR.

Disclaimers: None.

\section{REFERENCES}

Arbaje, A. I., J. L. Wolff, Q. Yu, N. R. Powe, G. F. Anderson, and C. Boult. 2008. "Postdischarge Environmental and Socioeconomic Factors and the Likelihood of Early Hospital Readmission among Community-Dwelling Medicare Beneficiaries." Gerontologist 48(4):495-504.

Area Resource File. 2009-2010. US Department of Health and Human Services, Health Resources and Services Administration, Bureau of Health Professions. Rockville, MD: ARF.

Bernheim, S. M., J. N. Grady, Z. Lin, Y. Wang, Y. Wang, S. V. Savage, K. R. Bhat, J. S. Ross, M. M. Desai, A. R. Merrill, L. F. Han, M. T. Rapp, E. E. Drye, S. L. Normand, and H. M. Krumholz. 2010. "National Patterns of Risk-Standardized Mortality and Readmission for Acute Myocardial Infarction and Heart Failure. Update on Publicly Reported Outcomes Measures Based on the 2010 Release.” Circulation: Cardiovascular Quality and Outcomes 3 (5): 459-67.

Bradley, E. H., J. Herrin, L. Curry, E. J. Cherlin, Y. Wang, T. R. Webster, E. E. Drye, S. L. Normand, and H. M. Krumholz. 2010. "Variation in Hospital Mortality Rates for Patients with Acute Myocardial Infarction.” American Journal of Cardiology 106 (8): 1108-12. doi:10.1016/j.amjcard.2010.06.014.

Bradley, E. H., J. Herrin, B. Elbel, R. L. McNamara, D. J. Magid, B. K. Nallamothu, Y. Wang, S. L. Normand, J. A. Spertus, and H. M. Krumholz. 2006. "Hospital Quality for Acute Myocardial Infarction: Correlation among Process Measures and 
Relationship with Short-Term Mortality.” Journal of the American Medical Association 296 (1): 72-8.

Dartmouth Atlas of Health Care. 2014. [accessed on January 22, 2014]. Available at http://www.dartmouthatlas.org/

Franks, P., and K. Fiscella. 2002. "Effect of Patient Socioeconomic Status on Physician Profiles for Prevention, Disease Management, and Diagnostic Testing Costs." Medical Care 40 (8): 717-24.

Guttman, L. 1953. "Image Theory for the Structure of Quantitative Variables.” Psychometrica 18: 277-96.

Howie-Esquivel, J., and J. G. Spicer. 2012. "Association of Partner Status and Disposition with Rehospitalization in Heart Failure Patients." American Journal of Critical Care 21(3):e65-73. doi:10.4037/ajcc2012382. PMID: 22549582.

Kaiser, H. F., and J. Rice. 1974. "Little Jiffy, Mark IV.” Educational and Psychological Measurement 34: 111-7.

Keating, N. L., M. B. Landrum, E. B. Lamont, S. R. Bozeman, and B. J. McNeil. 2012. "Area-Level Variations in Cancer Care and Outcomes." Medical Care 50 (5): 366-73.

Laskey, W., N. Spence, X. Zhao, R. Mayo, R. Taylor, C. P. Cannon, A. F. Hernandez, E. D. Peterson, and G. C. Fonarow. 2010. "Regional Differences in Quality of Care and Outcomes for the Treatment of Acute Coronary Syndromes: An Analysis from the Get with the Guidelines Coronary Artery Disease Program.” Critical Pathways in Cardiology 9 (1): 1-7.

Herrin, J., J. St Andre, K. Kenward, M. S. Joshi, A.-M. Audet, and S. J. Hines. 2015. "Community Factors and Hospital Readmission Rates." Health Services Research 50 (1): 20-39.

Home Health Compare. 2014. [accessed on September 28, 2012]. Available at http:// www.medicare.gov/HomeHealthCompare

Hospital Compare. 2014. [accessed on January 22, 2014]. Available at http://www.hospitalcompare.hhs.gov/

Li, Y., J. Yin, X. Cai, J. Temkin-Greener, and D. B. Mukamel. 2011. "Association of Race and Sites of Care with Pressure Ulcers in High-Risk Nursing Home Residents." Journal of the American Medical Association 306 (2): 179-86.

Lindenauer, P. K., T. Lagu, M. B. Rothberg, J. Avrunin, P. S. Pekow, Y. Wang, and H. M. Krumholz. 2013. "Income Inequality and 30-Day Outcomes after Acute Myocardial Infarction, Heart Failure, and Pneumonia: Retrospective Cohort Study." British Medical Journal346: f521.

Marcantonio, E. R., S. McKean, M. Goldfinger, S. Kleefield, M. Yurkofsky, and T. A. Brennan. 1999. "Factors Associated with Unplanned Hospital Readmission among Patients 65 Years of Age and Older in a Medicare Managed Care Plan.” American Journal of Medicine 107(1):13-7.

Mor, V., J. Zinn, J. Angelelli, J. M. Teno, and S. C. Miller. 2004. "Driven to Tiers: Socioeconomic and Racial Disparities in the Quality of Nursing Home Care." Milbank Quarterly 82(2):227-56.

Nunnally, J. C., and I. H. Bernstein. 1994. Psychometric Theory. 3rd edition. New York: McGraw-Hill. 
Nursing Home Compare. 2014. [accessed on September 28, 2012]. Available at http:// www.medicare.gov/NursingHomeCompare/

Pop Facts. 2010. Pop-Facts Premier. 2010.1 Nielsen Demographic Update.

Rosati, R. J., L. Huang, M. Navaie-Waliser, and P. H. Feldman. 2003. "Risk Factors for Repeated Hospitalizations among Home Healthcare Recipients.” Journal for Healthcare Quality 25 (2): 4-10.

Shulan, M., K. Gao, and C. D. Moore. 2013. "Predicting 30-Day All-Cause Hospital Readmissions." Health Care Management Sciences 16(2):167-75.

Smith, D. B., Z. Feng, M. L. Fennell, J. Zinn, and V. Mor. 2008. "Racial Disparities in Access to Long-Term Care: The Illusive Pursuit of Equity." Journal of Health Politics, Policy and Law 33 (5): 861-81.

Thomas, K. 2014. "Medicare Star Ratings Allow Nursing Homes to Game the System." New York Times. Published: Aug. 24.

Tsuchihashi, M., H. Tsutsui, K. Kodama, F. Kasagi, S. Setoguchi, M. Mohr, T. Kubota, and A. Takeshita. 2001. "Medical and Socioenvironmental Predictors of Hospital Readmission in Patients with Congestive Heart Failure." American Heart Journal 142(4):E7.

VanLare, J. M., and P. H. Conway. 2012. "Value-Based Purchasing-National Programs to Move from Volume to Value." New England Journal of Medicine 367: 292-5.

Werner, R. M., and E. T. Bradlow. 2006. "Relationship between Medicare's Hospital Compare Performance Measures and Mortality Rates" Journal of the American Medical Association 296 (22): 2694-702. PMID:17164455.

\section{SUPPORTING INFORMATION}

Additional supporting information may be found in the online version of this article:

Appendix SA1: Author Matrix. 